\title{
LA REVISIÓN JUDICIAL DE LAS MEDIDAS LIMITATIVAS DE DERECHOS EN EL MARCO DE LA COVID-19
}

\section{The Judicial Review of the Limiting Measures of Rights in the Framework of COVID-19}

\author{
Antonio Jesús Alonso Timón \\ Universidad Pontificia Comillas \\ E-mail: atimon@icade.comillas.edu
}

La revisión judicial de las decisiones adoptadas por las autoridades sanitarias con el objetivo de luchar contra la pandemia de la COVID-19 cobran una relevancia notable desde el punto de vista de la protección de los derechos fundamentales de los ciudadanos y del respeto a principios nucleares del Estado de Derecho como es la separación de poderes. El examen de legalidad y de proporcionalidad que los tribunales están obligados a realizar supone una garantía frente a las tentaciones autoritarias. Quizás por eso, para salvar estos controles jurisdiccionales, se haya optado por la aplicación de unas herramientas de excepcionalidad constitucional que minoran los derechos fundamentales de los ciudadanos con un control judicial mucho más debilitado. ¿Se entiende de qué va este juego?

The judicial review of the decisions adopted by the health authorities with the aim of fighting against the COVID-19 pandemic are of notable importance from the point of view of the protection of the fundamental rights of citizens and respect for the nuclear principles of the State of Law such as the separation of powers. The legality and proportionality test that the courts are obliged to carry out is a guarantee against authoritarian temptations. Perhaps that is why, in order to save these jurisdictional controls, it has been chosen to apply constitutional exception tools that undermine the fundamental rights of citizens with much more weakened judicial control. Is it understood what this game is about?

Estado de Derecho; control judicial; derechos fundamentales; principio de proporcionalidad Rule of law; judicial review; fundamental rights; principle of proportionality

Key words

(i) Resumen<smiles>O=CCCCCO</smiles>
Fechas 


\section{Introducción}

La pandemia ocasionada por el virus SARS-CoV-2, más conocido como coronavirus, ha removido los cimientos de nuestra vida y ha generado cambios importantes desde el punto de vista jurídico, político, social y económico.

Las respuestas que desde el poder público se han dado a esta pandemia han sido múltiples y se han plasmado en una batería de medidas jurídicas y políticas de notable calado desde que, a mediados del mes de marzo de 2020, se declaró el estado de alarma en España y se decretaron confinamientos severos por todo el continente europeo.

No había precedentes en el ordenamiento español desde la aprobación de la Constitución de 1978 de la adopción por parte de los poderes públicos de medidas tan restrictivas de derechos y libertades como las que se han ido adoptando a lo largo de este año 2020 en nuestro país con el objetivo de luchar contra esta pandemia. La única ocasión anterior en que el Gobierno, socialista también, utilizó el estado de alarma para acabar con una huelga salvaje de controladores aéreos en el año 2010 no es en modo alguno asimilable a la situación actual. Ni por la cantidad de días de duración de dicho estado de alarma ni por la calidad de las medidas adoptadas, que no tuvieron incidencia alguna en la vida cotidiana de la mayoría de los ciudadanos porque no afectaron al ejercicio de derechos fundamentales y libertades públicas de la misma manera en que ahora se han visto afectados.

Las opciones jurídicas para adoptar estas medidas limitativas pasaban por la utilización de dos herramientas diferentes. Una, el estado de alarma, regulado en el artículo 116 de la Constitución y desarrollado por la Ley Orgánica 4/1981, de 1 de junio, de los Estados de alarma, excepción y sitio. Concretamente, el artículo 4.b) de dicha Ley Orgánica, contempla el supuesto de hecho adecuado que habilitaría al Gobierno para decretar el estado de alarma, como así se ha hecho. Pero más allá de ese instrumento jurídico de carácter excepcional, existen otros mecanismos en la legislación ordinaria que se pueden utilizar para adoptar medidas de lucha contra la COVID-19. Nos referimos a la legislación sectorial sanitaria, que contiene normas aprovechables y efectivas, con ciertos retoques para adaptarlas a la situación actual, para que no se tenga que acudir con vocación de permanencia a herramientas como el estado de alarma, que no deberían prolongarse en el tiempo más de lo estrictamente imprescindible.

En efecto, tanto la Ley Orgánica 3/1986, de 14 de abril, de medidas especiales en materia de salud pública como la Ley 16/2003, de 28 de mayo, de cohesión y calidad del Sistema Nacional de Salud habilitan a los poderes públicos para la adopción de medidas de lucha contra crisis sanitarias como la que nos está tocando vivir y son herramientas de legalidad ordinaria que, con distintos matices y limitaciones, evitan tener que acudir a herramientas de excepcionalidad que, por tiempo prolongado en su uso, erosionan nuestro sistema democrático al otorgar al poder ejecutivo un incremento de poderes quasi omnímodos y reducirse el control de su ejercicio tanto por parte del poder legislativo como por parte del poder judicial, como hemos tenido ocasión de comprobar recientemente y pondremos de manifiesto a lo largo de este trabajo.

La adopción de medidas de lucha contra la pandemia desde el prisma de la citada legislación ordinaria ha tenido una vida efímera en nuestro país. Se acudió a ella en el periodo que medió entre la mal llamada "desescalada" (término delirante) y el comienzo de la también denominada "segunda ola". Es decir, entre los meses de julio y octubre de 2020, hasta que, de nuevo, con grandilocuencia y con escaso respeto por la legalidad constitucional, el presidente del 
Gobierno anunciase el día 25 de octubre de 2020 que volvíamos a un estado de alarma en todo el territorio nacional, salvo Canarias, por un periodo, ni más ni menos, que de seis meses (hasta el 9 de mayo de 2021). Ello demuestra el jaez no solo del ejecutivo central, sino de los diferentes ejecutivos autonómicos que se lanzaron a solicitar el estado de alarma al presidente del Gobierno para no tener que adoptar medidas que pudieran ser "tumbadas" en sede jurisdiccional. El ejecutivo nacional en este segundo estado de alarma se ha puesto de perfil ante la caótica e incompetente gestión que el mando único realizó durante los meses iniciales de la pandemia, delegando la gestión en los presidentes autonómicos. Por su parte, los ejecutivos autonómicos han renunciado a solicitar del Gobierno central la modificación de la legislación ordinaria para actualizarla y adaptarla a la situación actual y han preferido vivir política y jurídicamente instalados en el poder plenipotenciario que la excepcionalidad les otorga. A ello ha contribuido el revolcón que en numerosas ocasiones los tribunales de justicia han dado a algunas insensatas medidas adoptadas por los diferentes Gobiernos autonómicos y locales. De nada ha servido la reforma exprés tramitada durante el mes de septiembre en el parlamento nacional para agilizar los plazos de autorización o ratificación de las medidas adoptadas por los Gobiernos y la atribución de dicha competencia a los Tribunales Superiores de Justicia (para medidas autonómicas y locales) y a la Audiencia Nacional (para medidas estatales) en aras de dotar de una mayor seguridad jurídica y homogeneidad a las respuestas judiciales. Al revés, dicha reforma ha servido para aumentar el ego y la soberbia de los poderes ejecutivos, que no han soportado que los tribunales les sacasen los colores en alguna de las resoluciones emitidas en octubre de 2020. Lejos de seguir los consejos dados por nuestra jurisprudencia y las iniciativas surgidas en el seno de la oposición en el sentido de reformar la legislación ordinaria sanitaria para adaptar las medidas a adoptar a la situación actual, el Gobierno de la nación, con la connivencia de la mayoría de los ejecutivos autonómicos ha preferido profundizar en una deriva autoritaria que erosiona el sistema de contrapesos de poder, evitando, de manera clara y dudosamente constitucional, los controles parlamentarios y judiciales al apostar por un estado de alarma tan prolongado e ineficaz (por delegarlo en las comunidades autónomas, lo que profundizará en el caos de gestión) como irrespetuoso con las sugerencias que desde distintas instancias internacionales (Unión Europea y Consejo de Europa) se han hecho para evitar acudir a herramientas tan excepcionales y restrictivas como esta.

Iremos desgranando todos estos asuntos a lo largo de este trabajo, poniendo de manifiesto cuáles han sido los pronunciamientos judiciales más relevantes en el control de las medidas adoptadas por los diferentes poderes ejecutivos territoriales y la doctrina jurisprudencial que en ellas se contiene.

\section{Jurisprudencia destacable a lo largo de estos meses}

No podemos, desgraciadamente, analizar con detenimiento en este apartado muchos pronunciamientos jurisprudenciales constitucionales. Y ello, por dos motivos. Primero, porque el Tribunal Constitucional no ha tenido a bien pronunciarse todavía sobre los dos recursos de inconstitucionalidad planteados contra los dos Reales Decretos de declaración de estado de alarma del año 2020, a pesar de que el primero de ellos se interpuso hace casi ya un año y que, desde luego, el silencio del Alto Tribunal ha provocado ya una más que sobrada desaparición del objeto del recurso, pues el primer estado de alarma frente al que se interpuso finalizó a principios del mes de junio de 2020. Segundo, porque la única Sentencia del Tribunal Constitucional que se ha dictado sobre el estado de alarma en nuestro país no nos sirve a los fines de este trabajo por haber sido dictada en un supuesto de hecho muy distinto, con una proble- 
mática muy diferente y sobre unas medidas que nada o poco tienen que ver con las que se han adoptado en los dos estados de alarma decretados por el Gobierno recientemente. Nos referimos a la Sentencia 83/2016, de 28 de abril.

No obstante, hay algunas cuestiones de dicha sentencia que debemos tener presentes y que son extrapolables a la situación actual.

La primera cuestión destacable y digna de ser tenida en cuenta de dicho pronunciamiento, es la doctrina acuñada en dicha sentencia sobre el alma de ley material que tienen los Reales Decretos de declaración de los estados de alarma, por lo que correspondería al Tribunal Constitucional y no a la jurisdicción ordinaria el control directo de los mismos, a pesar de ser normas que, en puridad, tienen cuerpo de reglamento. Y ello porque para prorrogar su aplicación se requiere la participación del Congreso, sin cuya autorización no puede desplegar efectos dicha situación de excepcionalidad constitucional (FJ 8 y 9$)^{1}$.

La segunda cuestión relevante es la referida a la suspensión de derechos fundamentales. En el mismo fundamento jurídico octavo, último párrafo, de ese pronunciamiento, el Tribunal Constitucional deja claro que, durante la vigencia del estado de alarma, por mor del artículo 55.1 de la Constitución, no se pueden suspender los derechos fundamentales, aunque sí limitarlos. Hubiese sido interesante que el Alto Tribunal, como era su obligación, se hubiera pronunciado sobre las restricciones a los derechos fundamentales impuestas durante la vigencia del primer estado de alarma, pues entendemos que vulneraban la Constitución al suponer una suspensión y no una mera limitación de derechos, cosa que no ocurre en el segundo estado de alarma, donde se ha decretado una limitación y no una suspensión completa de, por ejemplo, la movilidad.

Nos centraremos ya en la competencia de la jurisdicción contencioso-administrativa para autorizar o ratificar las medidas de restricción sanitarias adoptadas por las Administraciones Públicas territoriales. Tras la modificación sufrida por la Ley de la Jurisdicción Contencioso-Administrativa en septiembre de $2020^{2}$, la competencia para fiscalizar las medidas restrictivas individuales o sobre colectivos determinados y perfectamente identificables se atribuye a los juzgados provinciales de lo contencioso-administrativo (artículo 8.6, párrafo segundo), la autorización o ratificación de las medidas restrictivas cuando los destinatarios de estas "no estén identificados individualmente y sean adoptadas por autoridades no estatales está atribuida a los Tribunales Superiores de Justicia (artículos 10.8) y a la Audiencia Nacional cuando dichas limitaciones colectivas son adoptadas por autoridades estatales (artículo 11.1.i)”.

Debemos precisar que dichas autorizaciones o limitaciones por parte de la jurisdicción contencioso-administrativa son operativas y necesarias cuando no estamos bajo el paraguas del estado de alarma. Si estamos bajo la vigencia del estado de alarma, como se acaba de explicar, el control de las medidas restrictivas corresponde al Tribunal Constitucional. Es por ello por lo que el análisis de esta jurisprudencia se va a centrar en las resoluciones de la jurisdicción contencioso-administrativa que se han producido durante los meses en que no hemos vivido bajo el estado de alarma, centrándonos en dos pronunciamientos muy relevantes que adoptan

1 Sobre el rango normativo del Real Decreto de declaración del estado de alarma, véase también el Auto del Tribunal Constitucional 7/2012, de 13 de enero. 
soluciones diametralmente opuestas. Nos referimos a los Autos del TSJ de Madrid de 8 de octubre y del TSJ de Castilla y León de 9 de octubre de 2020.

La autorización o ratificación de estas medidas restrictivas de lucha contra la pandemia deben llevarse a cabo, tal y como se indica en el Auto del TSJ de Madrid de 8 de octubre de 2020, teniendo en cuenta dos claros parámetros de control. Debe consistir, en primer lugar, en un juicio de legalidad que analice si existe o no cobertura legal para adoptar dichas medidas limitativas cuya autorización o ratificación se solicita y, en segundo lugar, en un juicio de proporcionalidad, analizando la necesidad, adecuación y razonabilidad de tales medidas. Y, en todo caso, la finalidad que debe presidir tal fiscalización es la de no imponer sacrificios innecesarios para las libertades y derechos fundamentales.

Asimismo, es necesario puntualizar que la autorización o ratificación judicial será precisa, exclusivamente, para aquellas medidas sanitarias que impliquen la privación o restricción de la libertad o de otro derecho fundamental. El resto de las medidas sanitarias de lucha contra la pandemia no están necesitadas de autorización judicial, sin perjuicio, de su posible impugnación ante la jurisdicción contencioso-administrativa por los motivos generales de fiscalización (anulabilidad o nulidad, desviación de poder, etcétera).

Este control judicial tampoco se extiende a las recomendaciones sanitarias dirigidas a la población pero que no tienen fuerza imperativa.

Centrándonos ya en los dos pronunciamientos aludidos, el TSJ de Madrid, en su Auto de 8 de octubre de 2020 afirma que no existe cobertura legal de la norma invocada por la Comunidad de Madrid, en cumplimiento de la Orden comunicada del Ministerio de Sanidad para poder imponer la limitación de derechos pretendida en el territorio de dicha Comunidad ${ }^{3}$. Concretamente, el análisis se centra en el artículo 65 de la Ley 16/2003, de 28 de mayo, de cohesión y calidad del Sistema Nacional de Salud. El TSJ madrileño indica que aunque la naturaleza de Ley ordinaria no sería, a priori, un obstáculo para que la citada norma legal pudiera establecer limitaciones de los derechos fundamentales, siempre que no fueran restricciones directas o esenciales (que solo podrían imponerse por Ley Orgánica), no aprecia en la regulación que contiene el artículo 65 de la Ley 16/2003, de 28 de mayo, de cohesión y calidad del Sistema Nacional de Salud, habilitación legal alguna para el establecimiento de medidas limitativas del derecho fundamental a la libertad de desplazamiento y circulación de las personas (artículo 19 $\mathrm{CE}$ ), o de cualquier otro derecho fundamental. Ninguna mención, indica el TSJ de Madrid, se hace en el precepto, ya sea de forma directa o indirecta, a la posible limitación de derechos fundamentales con motivo del ejercicio de las funciones legalmente encomendadas al Consejo Interterritorial del Sistema Nacional de Salud. Y menos aún se establecen en forma alguna los presupuestos materiales de una eventual limitación de derechos fundamentales, inherentes a las más elementales exigencias de certeza y seguridad jurídica.

\footnotetext{
3 Auto 128/2020, de la Sección 8. a del TSJ de Madrid, que resuelve la petición formulada por el Letrado de la Comunidad de Madrid de fecha 5 de octubre de 2020 en la que se solicitaba la ratificación, al amparo del art. 10.8 de la LJCA, de la Orden 1273/2020, de 1 de octubre, de la Consejería de Sanidad de la Comunidad de Madrid, por la que se establecían medidas preventivas en determinados municipios de la Comunidad de Madrid en ejecución de la Orden del Ministro de Sanidad, de 30 de septiembre de 2020, por la que se aprueban actuaciones coordinadas en salud pública. En concreto, dicha orden, adoptada en el seno del Consejo Interterritorial del Sistema Nacional de Salud, afectaba a diez municipios de la Comunidad de Madrid: Alcalá de Henares, Alcobendas, Alcorcón, Fuenlabrada, Getafe, Leganés, Madrid, Móstoles, Parla y Torrejón de Ardoz.
} 
Por tanto, la ausencia de habilitación legal para la restricción del derecho fundamental en la norma expresada impediría su adopción con fundamento en la misma. Y ello sin perjuicio de la existencia en nuestro ordenamiento jurídico de otros instrumentos y procedimientos que autorizan, sin duda alguna, la limitación de los derechos fundamentales.

Para el TSJ de Madrid, la mera referencia a la declaración de actuaciones coordinadas en salud pública, encomendada al Consejo Interterritorial del Sistema Nacional de Salud y la mención de los supuestos y mecanismos previstos para la realización de tales actuaciones en el mencionado precepto legal, orientados básicamente a la coordinación y cooperación de las distintas Administraciones Públicas con competencias en materia de salud pública, no permiten identificar la imprescindible habilitación legal limitativa de derechos fundamentales que posibilitara la adopción de las medidas sanitarias con tal alcance.

Además, en dicho Auto, el TSJ de Madrid le recrimina al legislador estatal su inactividad legislativa para adaptar la normativa sectorial sanitaria a la pandemia actual, indicando que resulta llamativo que ante el escenario sanitario descrito no se abordara una reforma de nuestro marco normativo más acorde con las confesadas necesidades de combatir eficazmente la pandemia de la COVID-19.

Precisa igualmente el TSJ madrileño que, en ese Auto de 8 de octubre, se encuentra ante un escenario completamente diferente al contemplado en los procedimientos en los que dicha sala y sección se pronunciaron con anterioridad, ratificando las medidas sanitarias adoptadas en las Órdenes 1177/2020, 1178/2020 y 1226/2020, de la misma Consejería de Sanidad de la Comunidad de Madrid, pues en tales Órdenes el fundamento de sus decisiones sobre imposición de medidas sanitarias se encontraba en el concreto marco normativo que mencionaban e invocaban las aludidas Órdenes de la Consejería madrileña (Ley Orgánica de Medidas Especiales en materia de Salud Pública, Ley General de Sanidad, Ley General de Salud Pública y Ley de Ordenación Sanitaria de la Comunidad de Madrid) y eran fruto del propio ejercicio de sus competencias, y no, como aquí, mediando una Orden comunicada del Ministerio de Sanidad.

Este punto es esencial, a mi parecer, puesto que el TSJ deja claras dos cosas. Primero, que la norma de invocación es relevante (podría haberse invocado, como se hizo en los casos con los que compara este Auto, el artículo 3 de la Ley Orgánica 3/1986, de 14 de abril). Segundo, que el mero ejercicio de competencias coordinadas por parte del Ministerio de Sanidad en un órgano como el Consejo Interterritorial del Sistema Nacional de Salud al amparo del artículo 65 de la Ley 16/2003 o del artículo 151.2.a), párrafo segundo de la Ley 40/2015, de 1 de octubre, del Sector Público no es suficiente para la imposición automática de limitaciones a derechos fundamentales. Desde luego, coincidiendo con el pronunciamiento analizado, no parece de recibo que se puedan imponer medidas tan restrictivas como estas por un mero Acuerdo de un órgano administrativo de coordinación y cooperación multilateral como este. No se respetarían los estándares constitucionales de limitación de los derechos fundamentales, puesto que la limitación o restricción de los derechos fundamentales debe ser respetuosa con la reserva de ley prevista en los artículos 81.1 y 53.1 de la Constitución y cumplir con dos presupuestos de constitucionalidad: perseguir una finalidad constitucionalmente legítima y cumplir con el principio de proporcionalidad (Sentencia del Tribunal Constitucional 5/1981). No hay que olvidar que, por lo que respecta al instrumento legal hábil para establecer las limitaciones de los derechos fundamentales y en relación con la reserva de Ley orgánica del artículo 81.1, el Tribunal Constitucional viene acogiendo, desde la citada Sentencia 5/1981, de 13 de febrero, 
un criterio de interpretación estricto. Tanto en lo que se refiere al término a desarrollar como a la materia objeto de reserva.

Por todas estas consideraciones, el TSJ de Madrid procede a denegar en dicho Auto las medidas restrictivas solicitadas al entender que la norma invocada no respeta los límites constitucionales para la imposición de limitaciones al ejercicio de derechos fundamentales, lo que obligó al Gobierno a reaccionar rápidamente, declarando el estado de alarma circunscrito, única y exclusivamente, al territorio de la Comunidad de Madrid al día siguiente. Esta medida le daba ya la cobertura legal necesaria sin necesidad de ratificación judicial para poder imponer coercitivamente las medidas limitativas acordadas en el seno del Consejo Interterritorial del Sistema Nacional de Salud, aun con la posición en contra de la Comunidad de Madrid.

En sentido contrario a este pronunciamiento, el Auto del TSJ de Castilla y León, dictado tan solo un día después del que se acaba de analizar, ratifica las medidas limitativas de derechos adoptadas en relación con el municipio de León ${ }^{4}$.

El TSJ de Castilla y León indica que tanto el Estado (artículo 149.1.16 de la Constitución) como las comunidades autónomas (artículo 148.1.21) gozan de competencias en materia sanitaria para luchar contra esta pandemia y que esta actuación coordinada de las dos instancias territoriales (estatal y autonómica) es la que dio lugar a la adopción de estas medidas, que eran adecuadas para la protección de la salud y que se ajustaban a la normativa constitucional y ordinaria, ejerciendo sus propias competencias la comunidad autónoma de Castilla y León, sin discrepancia formal con el Estado, si bien previa coordinación por parte de la Administración del Estado, que también había ejercitado las competencias que le corresponden por razones de interés general para dar lugar a una actuación conjunta de las diversas comunidades autónomas.

La específica habilitación normativa para el ejercicio de las competencias requeridas se encuentra, en este caso, según el TSJ de Castilla y León, en el artículo 3 de la Ley 3/1986, de 14 de abril, de medidas especiales en materia de salud pública.

Como vemos, nos encontramos ante dos mundos distintos, ante dos pronunciamientos totalmente diferentes. Mientras el TSJ de Madrid no encuentra habilitación legal para la imposición de medidas limitativas de los derechos fundamentales a través de una actuación estatal coordinada en el seno del Consejo Interterritorial del Sistema Nacional de Salud, el TSJ de Castilla y León entiende que se cumplen los estándares constitucionales de limitación de los derechos fundamentales, residenciando la competencia estatal y autonómica en los artículos 149.1.16 y 148.1.21 y en el artículo 3 de la Ley Orgánica 3/1986. Pero lo cierto y verdad es que, como indica el TSJ de Madrid, dicho órgano de coordinación y cooperación no es competente para imponer unas medidas tan limitativas de derechos fundamentales por un mero Acuerdo adoptado en su seno no tener cobertura legal en la Ley 16/2003 para poder hacerlo.

\footnotetext{
4 Auto 245/2020 (Sala de Valladolid) por el que se resuelve la petición de ratificación presentada por el Letrado de la comunidad de Castilla y León, al amparo del art. 10.8 de la LJCA, de las medidas contenidas en la ORDEN SAN/1045/2020, de 6 de octubre, por la que se adoptan medidas sanitarias preventivas en el municipio de León, en ejecución de la Orden comunicada del Ministro de Sanidad, de 30 de septiembre de 2020, por la que se aprueban actuaciones coordinadas en salud pública, para responder ante situaciones de especial riesgo por transmisión no controlada de infecciones causadas por el SARS-CoV-2.
} 
Se necesita la participación de un órgano más cualificado que ese para mandarnos a todos a casa si queremos ser respetuosos con la ortodoxia constitucional.

La diferencia importante que existía en este caso es que la comunidad de Castilla y León estaba de acuerdo con la imposición de estas medidas limitativas en su territorio, mientras la Comunidad de Madrid se oponía a las mismas y apostaba por las que ella ya venía imponiendo con anterioridad. Sin embargo, este criterio político no debería ser el decisivo a la hora de ratificar las medidas limitativas de derechos por parte de los tribunales, cuyo examen debe quedar referido en estos casos, como ya se ha indicado, a examinar la cobertura legal y la proporcionalidad de las medidas y la verdad es que, coincidiendo con el TSJ de Madrid, no existía cobertura legal para imponer, vía Consejo Interterritorial, dichas medidas tan limitativas. No opina lo mismo el TSJ de Castilla y León, para quien el artículo 3 de la Ley Orgánica 3/1986 habilita para todo $y$, por tanto, para que cualquier órgano administrativo pueda dictar un Acuerdo por el que nos manden a todos a casa. Para justificar la cobertura legal del Acuerdo del Consejo Interterritorial del Sistema Nacional de Salud indica el citado tribunal que "ante la complejidad de la pandemia en la que nos encontramos, de difícil previsión por el legislador, se ha de entender que el tratamiento de esta grave epidemia se encuentra habilitada para su tratamiento en dicha Ley Orgánica, adaptando sus previsiones a la realidad actual”. Como se acaba de indicar, a diferencia del TSJ de Madrid, quien implora al legislador estatal una reforma urgente, el TSJ de Castilla y León acepta que el artículo 3 de la citada Ley Orgánica 3/1986 vale para todo, aunque reconoce la necesidad de adaptarlo a la realidad actual. Y, si hay que adaptarlo a la realidad actual, ¿por qué no se adapta por el cauce adecuado, que es su modificación legal?

No me gustaría finalizar este apartado sin hacer una referencia expresa a las sanciones impuestas durante la vigencia del primer estado de alarma. Entendemos, como ya pusimos de manifiesto en su momento, que eran ilegales por no respetar el principio de tipicidad.

Afortunadamente, dicha teoría ha sido corroborada por la mayoría de los pronunciamientos de los juzgados provinciales de lo contencioso-administrativo que han tenido ocasión de conocer los recursos interpuestos por los ciudadanos contra las mismas. Es cierto que en el segundo confinamiento se mitiga en cierta medida esta cuestión como consecuencia directa de la no suspensión de derechos fundamentales sino de su limitación, lo que es más respetuoso con la propia naturaleza del estado de alarma.

Sin embargo, no deja de llamar la atención la aplicación tan grosera que, desde el punto de vista jurídico, se hizo de la potestad sancionadora administrativa durante la vigencia del primer estado de alarma.

El mayor problema se daba con respecto a la aplicación práctica de la infracción administrativa de desobediencia. De acuerdo con los pronunciamientos jurisprudenciales que comentamos, los juzgados de lo contencioso-administrativo están precisando que no se puede imputar una infracción administrativa grave de desobediencia a la autoridad o a sus agentes por el genérico incumplimiento del ordenamiento jurídico cuando no ha existido un requerimiento expreso previo, personal y directo 5 .

\footnotetext{
5 Véanse las siguientes sentencias de los Juzgados provinciales de lo Contencioso Administrativo en relación con la imposición de sanciones durante el primer estado de alarma de 2020:

JCA número 1 de Córdoba (SJCA 2012/2020, de 4 de noviembre).

JCA número 3 de León (SJCA 160/2020, de 23 de noviembre).

JCA número 1 de Lugo (SJCA 2009/2020, de 19 de noviembre de 2020).
} 
El artículo 20 del Real Decreto 463/2020, de 14 de marzo, por el que se declara el estado de alarma indicaba, en relación con la potestad sancionadora, que el incumplimiento o la resistencia a las órdenes de las autoridades competentes en el estado de alarma será sancionado con arreglo a las leyes, en los términos establecidos en el artículo diez de la Ley Orgánica 4/1981, de 1 de junio. Dicha remisión era prácticamente una remisión en blanco, pues dicho precepto no añade nada nuevo ni tipifica infracción alguna. Tanto el artículo 10 como el 11 de la misma Ley Orgánica reguladora de los estados de alarma, excepción y sitio se limitan a describir una serie de medidas genéricas que pueden adoptar las autoridades bajo la vigencia del estado de alarma que, desde el punto de vista sancionador, serían incompatibles con la correcta aplicación del principio de tipicidad, que exige una descripción concreta de la conducta sancionable y, por ello, se estaba vulnerando este principio esencial del ejercicio de la potestad sancionadora, regulado como tal en el artículo 27 de la Ley 40/2015, de 1 de octubre, de Régimen Jurídico del Sector Público y exigido con rigor por nuestra jurisprudencia ${ }^{6}$.

Para cubrir ese vacío legal se acudió a la Ley Orgánica 4/2015, de 30 de marzo, de Protección de la Seguridad Ciudadana y, más concretamente, a su Capítulo V, que es el que regula el régimen sancionador. El problema es que la tipificación que de las infracciones leves, graves y muy graves se contiene en los artículos 35, 36 y 37 de dicha norma está cogido con pinzas para ser utilizado en la mayoría de los supuestos en que se ha utilizado para imponer sanciones derivadas del supuesto incumplimiento de las medidas adoptadas por el Gobierno durante la vigencia del estado de alarma. Y ello por dos motivos.

Primero, porque la aplicación práctica que del primer estado de alarma llevó a cabo el Gobierno excedía los límites constitucionales y legales del propio estado de alarma y, de hecho, en este ámbito concreto, se fue más allá de las previsiones contenidas en el propio Real Decreto $463 / 2020$.

Segundo, porque la descripción de los tipos que los citados artículos de la Ley de Seguridad Ciudadana de 2015 no están pensados para la mayoría de las situaciones que se han producido durante la vigencia del estado de alarma. Prueba de ello es que el propio Ministerio del Interior tuvo que elaborar circulares que explicasen a los agentes de la autoridad cómo interpretar las normas y cómo proceder a la hora de formular las denuncias porque no estaba claro, en modo alguno, que las conductas de la mayoría de los ciudadanos fuesen subsumibles en el tipo de infracciones donde se les había situado de manera forzada y un tanto arbitraria.

Lo verdaderamente cierto es que, en la mayoría de los casos, no existía ni incumplimiento ni resistencia en la conducta de los ciudadanos, por lo que no cabría amparar esas sanciones en lo establecido en el artículo 10 de la Ley Orgánica 4/1981, como, afortunadamente, los jueces de lo contencioso se están encargando de confirmar ahora.

JCA número 3 de Oviedo (SJCA 2003/2020, de 9 de noviembre de 2020).

JCA número 3 de Pamplona (SJCA 1967/2020, de 5 de octubre de 2020).

JCA número 1 de Vigo (SJCA 1816/2020, de 19 de octubre de 2020).

6 Véanse las Sentencias del Tribunal Constitucional 61/1990, de 29 de marzo y del Tribunal Supremo de 5 de febrero de 1990. 


\section{Conclusiones}

- La autorización o ratificación judicial de las medidas restrictivas de derechos fundamentales adoptadas por las autoridades sanitarias de los tres niveles administrativos territoriales está atribuida a la jurisdicción contencioso-administrativa cuando no nos encontramos bajo la vigencia de un estado de alarma. Bajo la vigencia del estado de alarma corresponde al Tribunal Constitucional la supervisión de la restricción de derechos fundamentales, al tener el Real Decreto de declaración del estado de alarma valor de ley material por la necesaria intervención posterior del Congreso para la prórroga de este, de acuerdo con la doctrina del Tribunal Constitucional.

- La autorización o ratificación de las medidas sanitarias atribuida a la jurisdicción contencioso-administrativa cuando se adoptan sin el paraguas del estado de alarma se tramita por el procedimiento preferente especial regulado en el nuevo artículo 122quater de la Ley de la Jurisdicción Contencioso-Administrativa cuando se restringen derechos fundamentales. Cualquier otra medida sanitaria de lucha contra la pandemia que no implique restricción de derechos fundamentales es fiscalizada por la jurisdicción contencioso-administrativa o por cualquier otro orden jurisdiccional en los términos generales en que la Constitución, en su artículo 106.1, regula el control y fiscalización de las actuaciones administrativas por parte de los tribunales de justicia.

- El control judicial de las medidas restrictivas de derechos fundamentales adoptadas por las autoridades sanitarias debe quedar circunscrito a dos ámbitos: control de legalidad, referido a la cobertura legal de dichas medidas, y control de proporcionalidad, residenciado en el análisis de la necesidad, adecuación y razonabilidad de las medidas propuestas.

- La limitación de derechos fundamentales debe tener cobertura de Ley orgánica o de Ley ordinaria cuando dicha limitación por Ley ordinaria se lleva a cabo en desarrollo de una Ley orgánica.

- El artículo 3 de la Ley Orgánica 3/1986, de 14 de abril, de medidas especiales en materia de salud pública, habilita a las autoridades sanitarias a adoptar medidas de limitación de derechos fundamentales, pero sería necesaria, como ha puesto de manifiesto parte de la doctrina, alguna jurisprudencia y ha propuesto la oposición por medio de una proposición de Ley, una reforma de dicho artículo para adaptarlo a la lucha contra la pandemia actual, concretando las medidas restrictivas que las Administraciones Públicas pueden adoptar con el objetivo de conseguir una mayor eficiencia y, sobre todo, una mayor seguridad jurídica.

- Ni el Consejo Interterritorial del Sistema Nacional de Salud es órgano idóneo para imponer restricciones de derechos fundamentales ni la Ley 16/2003, de 28 de mayo, de Cohesión y Calidad del Sistema Nacional de Salud, otorga cobertura legal a dicho órgano de cooperación multilateral para imponer tales limitaciones.

- La mayor parte de las sanciones impuestas a los ciudadanos durante el primer estado de alarma decretado por el Real Decreto 463/2020 son ilegales por no respetar el principio de tipicidad y aplicar tipos infractores por analogía, lo que está prohibido por el artículo 27.4 de la Ley 40/2015, como está poniendo de manifiesto la jurisprudencia. Especial- 
mente grave es el supuesto de sanciones por desobediencia, pues en la inmensa mayoría de los casos dicha desobediencia no se produjo, ya que, para que exista, como se están encargando de recordar los jueces de lo contencioso-administrativo, no basta un genérico incumplimiento del ordenamiento jurídico, sino que es necesario que haya existido un requerimiento expreso previo, personal y directo. 